\title{
"A dor e a delícia" do internato de atenção primária em saúde: desafios e tensões
}

\author{
"The pain and the delight" of primary health \\ care internship: challenges and tensions \\ Daniela Fontes Bezerra ${ }^{1}$, Fernando Adami ${ }^{1}$, Lígia de Fátima Nobrega Reato ${ }^{1}$, Marco Akerman \\ ${ }^{1}$ Curso de Medicina, Faculdade de Medicina do ABC (FMABC) - Santo André (SP), Brasil.
}

DOI: http://dx.doi.org/10.7322/abcshs.v40i3.790

\begin{abstract}
RESUMO
Introdução: As Diretrizes Curriculares Nacionais para o curso de Medicina sinalizaram a necessidade de formar um médico capaz de atuar conforme as necessidades de saúde da população visando o fortalecimento do Sistema Único de Saúde. A implantação de um estágio, na área de Atenção Primaria à saúde, na Faculdade de Medicina $A B C$, resultou em uma reforma mais ampliada do internato médico. Objetivo: Identificar a percepção dos estudantes quanto a inserção do ciclo de Atenção Primária à Saúde (CAPS) no internato médico. Métodos: Estudo transversal que utilizou um questionário fechado, aplicados aos estudantes do $5^{\circ}$ e $6^{\circ}$ anos, totalizando 157 participantes, em 2011 abordando os aspectos teóricos e práticos do CAPS. Resultados: Chamou a atenção na comparação entre o $5^{\circ}$ e $6^{\circ}$ anos do Ciclo de Atenção Primaria à Saúde: o CAPS no $5^{\circ}$ ano teve o maior número de quesitos respondidos com regular e fraco, enquanto que o $6^{\circ}$ ano teve um grande número respondido com ótimo e bom. Observaram-se diferenças marcantes quanto à percepção dos estudantes entre os aspectos abordados do CAPS entre os estudantes do $5^{\circ}$ e $6^{\circ}$, com destaque para maior grau de satisfação com o $6^{\circ}$ ano (UBS tradicional) do que com o $5^{\circ}$ ano Estratégia de saúde da família (ESF). Conclusão: Há indícios de que os respondentes validaram a implementação do CAPS, mas ainda restam desafios e tensões quanto à estruturação e integração ensino e serviço.
\end{abstract}

Palavras-chave: educação médica; serviços de integração docente-assistencial; internato e residência.

\begin{abstract}
Introduction: The National Curriculum Guidelines for the medical school showed that a doctor to graduate is necessary to be able to act upon the health needs of the population aimed at strengthening the Unified Health System. The implementation of a stage in Primary Care area of health, ABC School of Medicine, has resulted in a broader reform of the medical internship. Objective: To identify the perception of students and the insertion of the Primary Health Care Cycle (CAPS) in medical internship. Methods: Cross-sectional study that used a closed questionnaire applied to students of 5th and 6th year, totaling 157 participants in 2011 addressing the theoretical and practical aspects of CAPS. Results: They draw attention in the comparison between the 5th and 6th year of Primary Care Cycle Health: CAPS at 5 years had the highest number of answered questions with regular and weak, while the $6^{\text {th }}$ grade had a large number responded with great and good. There were marked differences in the perception of students among the topics of CAPS aspects among students of $5^{\text {th }}$ and $6^{\text {th }}$, highlighting greater degree of satisfaction with the $6^{\text {th }}$ grade (Traditional UBS) than with the 5 year Family Health Strategy (FHS). Conclusion: There are indications that respondents validated the implementation of CAPS, but there are still challenges and tensions on the structuring and integrating teaching and service.
\end{abstract}

Keywords: education, medical; teaching care integration services; internship and residency. 


\section{INTRODUÇÃO}

Como forma de garantir o acesso à saúde universal e gratuita a todos, o Estado Brasileiro instituiu pela Constituição Federal, em 1988, o Sistema Único de Saúde (SUS). O SUS busca permitir acesso à saúde, financiado com recursos provenientes da União, dos estados e municípios. A fim de se prover profissionais capazes de atuarem em consonância com nos princípios da Universalidade, Integralidade, Equidade, Regionalização e a Hierarquização da rede, das ações e dos serviços de saúde ${ }^{1}$, precisava-se construir um novo modelo de ensino de acordo com as necessidades da população brasileira. A partir de então, passou a ser competência do SUS atuar na formação de recursos humanos.

Nesse sistema, a saúde deixou de ser sinônimo apenas de cura de agravos, onde a preocupação era a doença e o tratamento, para ter um sentido muito mais amplo, incluindo prevenção de agravos e promoção da saúde ${ }^{1}$. Para que isso possa se concretizar, os profissionais atuantes precisam ter um olhar mais sensível, pois, além dos agravos, a saúde deve estar correlacionada à qualidade de vida da população, deixando de ser ausência de doença para ser "medida" através de fatores como alimentação, moradia, lazer, trabalho, educação, saneamento básico, entre outros.

Como defende Starfield ${ }^{2}$, a atenção primária é um nível de um sistema de serviço de saúde que acolhe novas necessidades e problemas. Além disso, aborda os problemas mais comuns na comunidade, oferecendo serviços de prevenção, cura e reabilitação para maximizar a saúde e o bem-estar.

Segundo Franco et al. ${ }^{3}$, o dia a dia do médico está pautado em prescrições numa relação profissional-usuário, restrita ao campo biológico e influenciado por um complexo médico-industrial do século $\mathrm{XX}$, que tem entre outros aspectos recursos caro de diagnóstico e tratamento.

Na reorganização do modelo de assistência e na formação de profissionais o Ministério da Educação aprovou as Diretrizes Curriculares Nacionais (DCN), em outubro de 2001, com propostas curriculares a inserção do estudante de medicina no SUS, focando num atendimento primário e secundário. Com essa normativa, ficou determinado que o internato fosse reestruturado em clínica medica, clínica cirúrgica, pediatria e ginecologia e obstetrícia e na área da saúde coletiva ${ }^{4}$.

Inicia-se, na política nacional brasileira, conteúdos curriculares diversos e a possibilidade de construção de projetos políticos-pedagógicos (PPP) ${ }^{5}$. No ensino de graduação em Medicina, de acordo com a DCN, o perfil esperado do formando egresso/profissional é o de um médico com formação generalista, humanista, crítica e reflexiva, capacitado a atuar pautado em princípios éticos no processo de saúde-doença em seus diferentes níveis de atenção, com ações de promoção, prevenção, recuperação e reabilitação à saúde, na perspectiva da integralidade da assistência, com senso de responsabilidade social e compromisso com a cidadania, como promotor da saúde integral do ser humano.

Segundo Ferreira et al. ${ }^{6}$, a integralidade, como princípio do SUS, deve ser a base da reorganização da clínica do cuidado, com três conceitos fundamentais: clínica ampliada, sistema de cogestão e a integração dos diversos pontos de conexão de um sistema público de saúde.

A clínica ampliada tem como objetivo a responsabilidade singular, vínculo estável entre a equipe de saúde e paciente, tendo como foco o profissional referência, ponto importante na construção da clínica de cuidado.

Ceccim e Feuerwerker ${ }^{7}$ discutem a integralidade da atenção no envolvimento da compreensão de noção da ampliação da clínica, o conhecimento sobre a realidade e o trabalho em equipe multiprofissional e transdisciplinar. Para eles, a imagem do conceito de quadrilátero da formação para a área de saúde (ensino, gestão, atenção e controle social) "serve à construção e organização de uma gestão da educação na saúde integrante da gestão do sistema de saúde, redimensionando a imagem dos serviços como gestão e atenção em saúde, valorizando o controle social".

A cogestão, entre profissionais e usuários da rede de saúde e a integração dos diversos pontos de conexão do SUS tensionam o modelo biomédico, onde os profissionais são capazes de planejar as medidas mais adequadas para a saúde coletiva e individual, dentro de uma ótica da clínica mais participativa.

"A finalidade de qualquer ação em saúde é produzir cuidado" em qualquer nível de atenção.

Por muitos anos, o modelo de ensino era centrado na passagem de informação, e o principal cenário de pratica era o hospital. Com esse novo modelo de assistência à construção de um projeto pedagógico, elaborado coletivamente, centrado no aluno como sujeito de aprendizagem, apoiado no professor como facilitador e mediador do processo ensino-aprendizagem, e com novos cenários de prática, se torna um desafio. Tal projeto deve buscar a formação integral e adequada do estudante por meio da articulação entre o ensino, a pesquisa e a extensão/assistência.

A partir das DCN, o Ministério da Saúde e o Ministério da Educação desencadearam várias políticas indutoras para que essas diretrizes fossem implementadas, como o Promed, Pró-Saúde. O Programa Nacional de Reorientação da Formação Profissional em Saúde tem como objetivo a integração ensino e serviço visando a formação de profissionais que atuam na área da saúde com uma abordagem integral do processo de saúde e doença com ênfase na Atenção Básica e assim mudando a organização do cuidado em saúde, que está historicamente centrada na doença e no atendimento hospitalar9. O Pró-Saúde promoveu e impulsionou as transformações na grade curricular do curso Medico na Faculdade de Medicina ABC. A valorização da atenção básica dentro da relação ensino-aprendizagem trouxe uma reestruturação do internato na Faculdade de Medicina do ABC, que, em 2009 , teve a criação do quinto ciclo, no $5^{\circ}$ e $6^{\circ}$ anos, somando-se aos outros quatro ciclos existentes: Clínica Médica, Clínica Cirúrgica, Pediatria e Ginecologia e Obstetrícia. O quinto ciclo correspondente à Atenção Primária à Saúde (CAPS).

Tais mudanças, focadas na formação de um profissional capaz de reconhecer e trabalhar as necessidades da população segundo 
as diretrizes do SUS, são consideradas uma estratégia pedagógica importante para a atividade prática da abordagem em Atenção Primária à Saúde. Desde então, fez-se necessário um reconhecimento, com o objetivo de analisar o aproveitamento da mudança no internato.

Com o aluno como ponto central do processo ensino-aprendizagem, esta pesquisa teve como público-alvo os alunos do quinto e sexto ano do curso de Medicina da Faculdade de Medicina do $A B C$ e o seu objetivo geral era identificar a percepção desses alunos quanto a inserção do CAPS no internato médico. O objetivo específico buscava de uma maneira comparativa com os ciclos tradicionais de clínica médica, clínica cirúrgica, pediatria, e ginecologia e obstetrícia, para que o aluno pudesse validar essa mudança no internato curricular.

\section{MÉTODOS}

Trata-se de um estudo transversal realizado na Faculdade de Medicina do ABC (FMABC), em Santo André (SP), com abordagem quantitativa, que utilizou um questionário, aplicado em dezembro de 2011 aos alunos do $5^{\circ}$ e $6^{\circ}$ anos.

O questionário era constituído por perguntas fechadas, que tinham as seguintes abordagens: aspectos teóricos do internato, aspectos práticos e qualidade geral do ciclo. Os Aspectos Teóricos abordados e os Aspectos Práticos avaliados são apresentados no Quadro 1.

O questionário foi baseado no Programa de Avaliação Curricular da Faculdade de Medicina da Universidade de São Paulo (USP), uma estratégia de avaliação usada desde 1989 na instituição ${ }^{10}$. O instrumento foi modificado buscando uma adaptação à realidade da FMABC.

Todos os alunos do $5^{\circ}$ e $6^{\circ}$ anos foram convidados a participar da pesquisa, haviam 97 alunos matriculados no $5^{\circ}$ ano e 99 alunos no $6^{\circ}$ ano, e responderem o questionário. Isso aconteceu na última semana de aula do ano de 2011.

Quadro 1: Quesitos abordados sobre os Aspectos Teóricos e Práticos

\begin{tabular}{|c|c|}
\hline Aspectos teóricos & Aspectos práticos \\
\hline $\begin{array}{l}\text { Esclarecimento adequado dos } \\
\text { objetivos do Ciclo de Atenção } \\
\text { Primária à Saúde }\end{array}$ & Relação preceptor e aluno \\
\hline $\begin{array}{l}\text { Utilização e distribuição do } \\
\text { tempo durante o ciclo }\end{array}$ & $\begin{array}{l}\text { Clientela atendida foi suficiente } \\
\text { para o aprendizado }\end{array}$ \\
\hline $\begin{array}{l}\text { Preocupação com o aprendizado } \\
\text { do aluno }\end{array}$ & Sua dedicação durante o ciclo \\
\hline $\begin{array}{l}\text { O estímulo ao raciocínio e } \\
\text { senso crítico }\end{array}$ & $\begin{array}{l}\text { Acompanhamento pelos } \\
\text { preceptores }\end{array}$ \\
\hline $\begin{array}{l}\text { Houve informações sobre o } \\
\text { programa do ciclo }\end{array}$ & $\begin{array}{l}\text { Orientação para a relação } \\
\text { médico paciente }\end{array}$ \\
\hline \multicolumn{2}{|l|}{ Correlação entre teoria e prática } \\
\hline \multicolumn{2}{|l|}{$\begin{array}{l}\text { Como foi o seu aprendizado } \\
\text { no ciclo }\end{array}$} \\
\hline $\begin{array}{l}\text { Qualidade do método de } \\
\text { avaliação }\end{array}$ & \\
\hline
\end{tabular}

O estudo foi aprovado pelo Comitê de Ética e pesquisa após submissão no programa plataforma Brasil. Número do Certificado de Apresentação para Apreciação Ética (CAAE): 11584312.0.0000.0082.

Foi oferecido aos alunos que decidiram participar da pesquisa um Termo de Livre Consentimento Esclarecido.

\section{RESULTADOS}

O questionário foi aplicado a 92 estudantes do quinto ano e 65 alunos do sexto ano do internato da FMABC, totalizando 157 participantes.

Quando se analisou os dados que falam sobre Aspectos Teóricos, Aspectos Práticos e Qualidade Geral do Ciclo de Atenção Primária à Saúde, temos os seguintes achados:

Em relação aos Aspectos Teóricos (Quadro 1), o resultado foi considerado pelos alunos do $5^{\circ}$ ano como "Regular + Fraco" nos seguintes quesitos - 63 alunos (68,5\%) no "esclarecimento adequado dos objetivos do ciclo", 68 alunos (74,4\%) "utilização e distribuição do tempo durante o ciclo", 63 alunos (68,5\%) "preocupação com o aprendizado do aluno", 63 alunos (68,5\%) "estímulo ao raciocínio e senso crítico", 53 alunos (57,6\%) "houve informações sobre o programa do ciclo", 55 alunos $(59,8 \%)$ "correlação entre teoria e prática" e $47(51,5 \%)$ dos alunos do $5^{\circ}$ ano avaliaram como "Ótimo + Bom" a "qualidade do método de avaliação".

Os alunos do $6^{\circ}$ ano consideraram "Ótimo + Bom" em todos os quesitos dos aspectos teóricos; 48 alunos (75,0\%) no "esclarecimento adequado dos objetivos do ciclo", 46 alunos (70,3\%) "utilização e distribuição do tempo durante o ciclo", 46 alunos (70,3\%) "preocupação com o aprendizado do aluno", 47 (73\%) "estímulo ao raciocínio e senso crítico", 53 (81,25\%) "houve informações sobre o programa do ciclo", 56 (85,5\%) “correlação entre teoria e prática" e 57 (88\%) "a qualidade do método de avaliação" (Figura 1).

Em relação aos Aspectos Práticos (Quadro 1), no $5^{\circ}$ ano, somente 2 quesitos tiveram resultado "Regular + Fraco" como mais frequente que foram a "relação preceptor e aluno" com $65(71,1 \%)$, e se "clientela atendida foi suficiente para o aprendizado" com 52 (56,7\%). Todos os outros quesitos tiveram como resposta "Ótimo + Bom", sendo a "dedicação durante o ciclo" de 63 (68,5\%), "acompanhamento pelos preceptores" $47(51,11 \%)$ e "orientação para a relação médico e paciente" $63(68,9 \%)$. No $6^{\circ}$ ano, todos os quesitos tiveram resultados "Ótimo + Bom", sendo a "relação preceptor e aluno" com 59 (90,5\%), se "clientela atendida foi suficiente para o aprendizado" com 56 (85,71\%), "dedicação durante o ciclo" de 57 (87,3\%), "acompanhamento pelos preceptores" 52 (79,4\%) e “orientação para a relação médico e paciente" 59 (91\%) (Figura 2).

Buscando uma percepção quanto a inserção do CAPS no internato numa visão comparativa com os outros ciclos; Clínica Medica, Clínica Cirúrgica, Pediatria e Ginecologia Obstetrícia, temos a Figura 3. O quesito qualidade geral do ciclo foi destacado por oferecer uma percepção mais abrangente do ciclo, e, neste aspecto, os alunos $5^{\circ}$ ano consideraram Clínica Médica com "Ótimo 


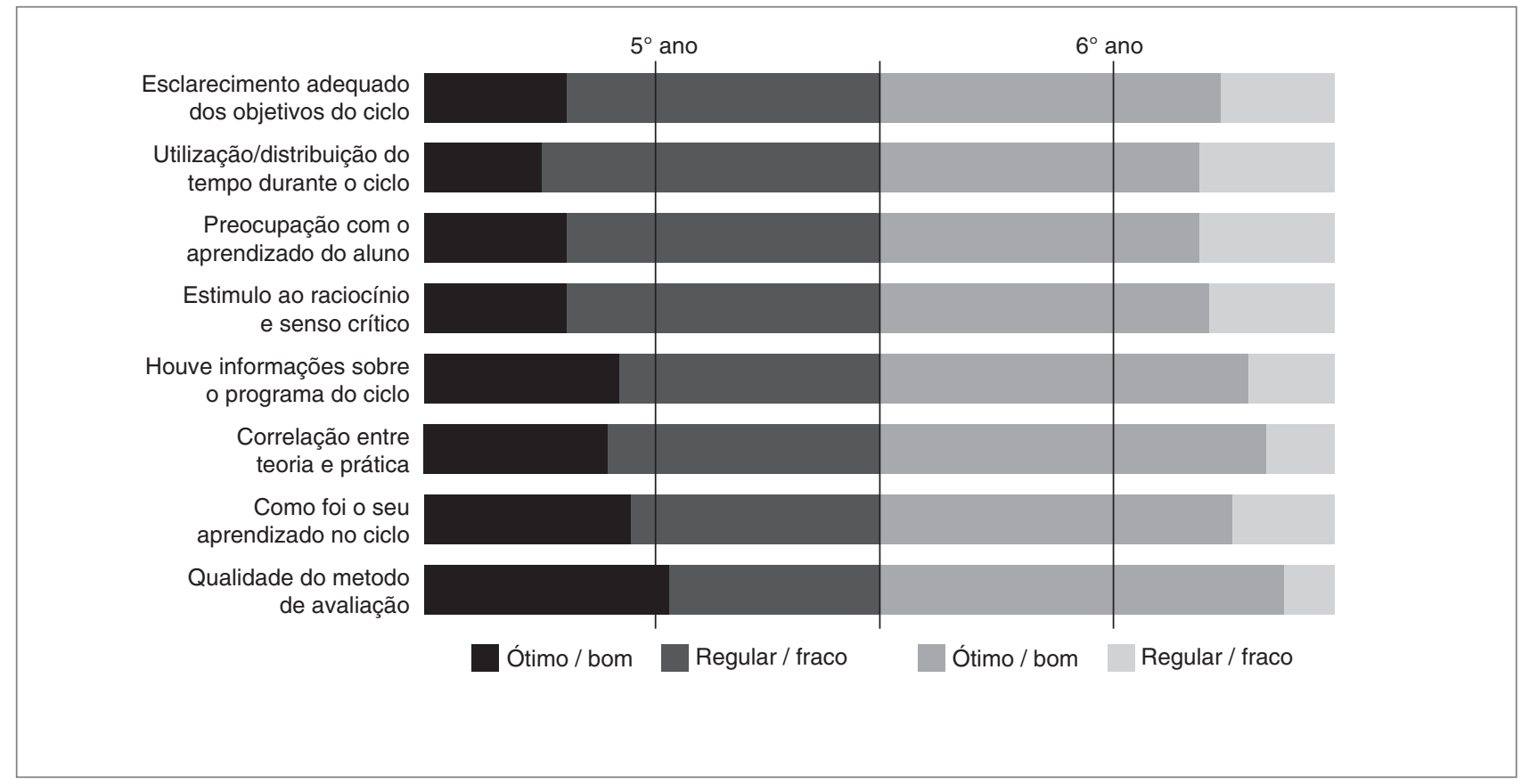

Figura 1: Matriz geral de avaliação do internato médico da Faculdade de Medicina do ABC, focando aspectos teóricos

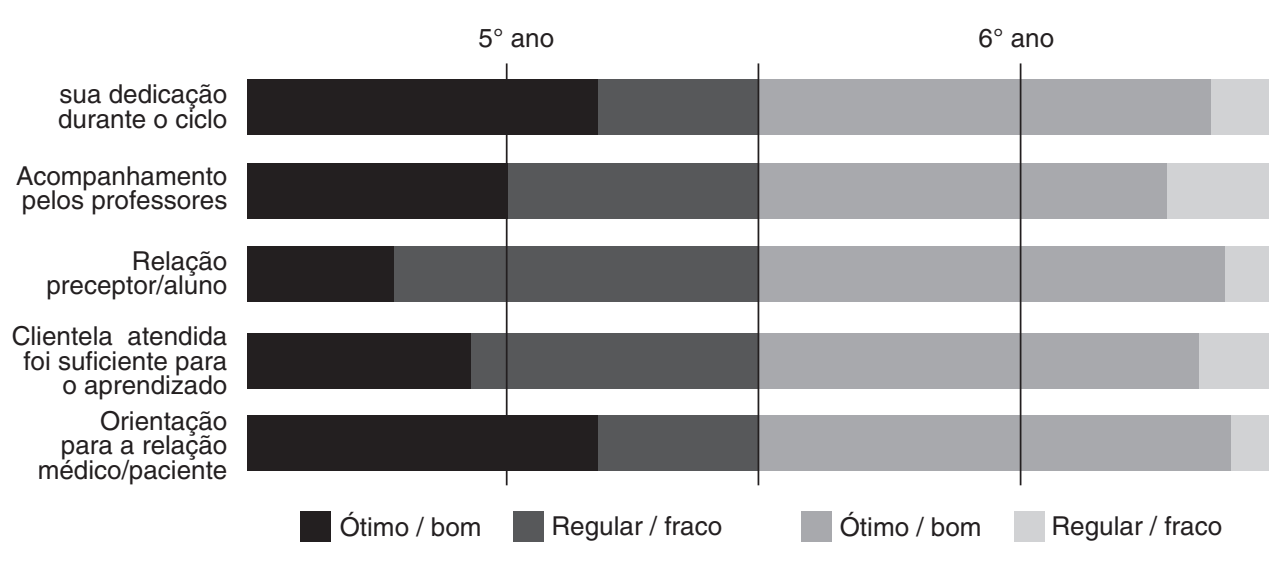

Figura 2: Matriz geral de avaliação do internato médico da Faculdade de Medicina do ABC, focando aspectos práticos

+ Bom” em 62 (67,4\%), Clínica Cirúrgica com “Ótimo + Bom” em 53 (57,6\%), Pediatria “Ótimo + Bom” em 79 (85,9\%), Ginecologia e Obstetrícia “Ótimo + Bom” em 56 (60,9\%), e o CAPS “Regular + Fraco" em 66 (71,7\%). No 60 ano, 36 (54,8\%) alunos consideraram a qualidade geral do ciclo de Clinica Médica como "Ótimo + Bom", Clínica cirúrgica como "Regular + Fraco" em 35 (53,2\%), Pediatria "Ótimo + Bom" em 64 (98,4\%), Ginecologia e Obstetrícia “Ótimo + Bom” em 60 (91,94\%) e CAPS com "Ótimo + Bom” em 52 (80,6\%) (Figura 3).

De acordo com os resultados focados na percepção do Ciclo de Atenção Primária à Saúde e sua inserção no internato médico, os respondentes consideraram necessária e importante à inserção do CAPS.

Do ponto de vista institucional, a diferença apontada nos resultados precisa ser aprofundada, pois o Ciclo de Atenção à
Saúde tem características peculiares no internato de medicina. No quinto ano, o cenário de prática é a estratégia de saúde da família e, no sexto, o foco são as atividades em uma Unidade Básica de Saúde (UBS), tradicional, que não tem estratégia de saúde da família.

\section{DISCUSSÃO}

O internato de Atenção Primária à Saúde na FMABC em 2011 tinha como características diferentes. No estágio no $5^{\circ}$ ano da FMABC, o CAPS possui a duração de dez semanas, sendo um período preparatório de uma semana e nove semanas no cenário de prática; já no $6^{\circ}$ ano, tem 8 semanas. Essa divisão se faz de maneira igual para todos os ciclos de internato. 


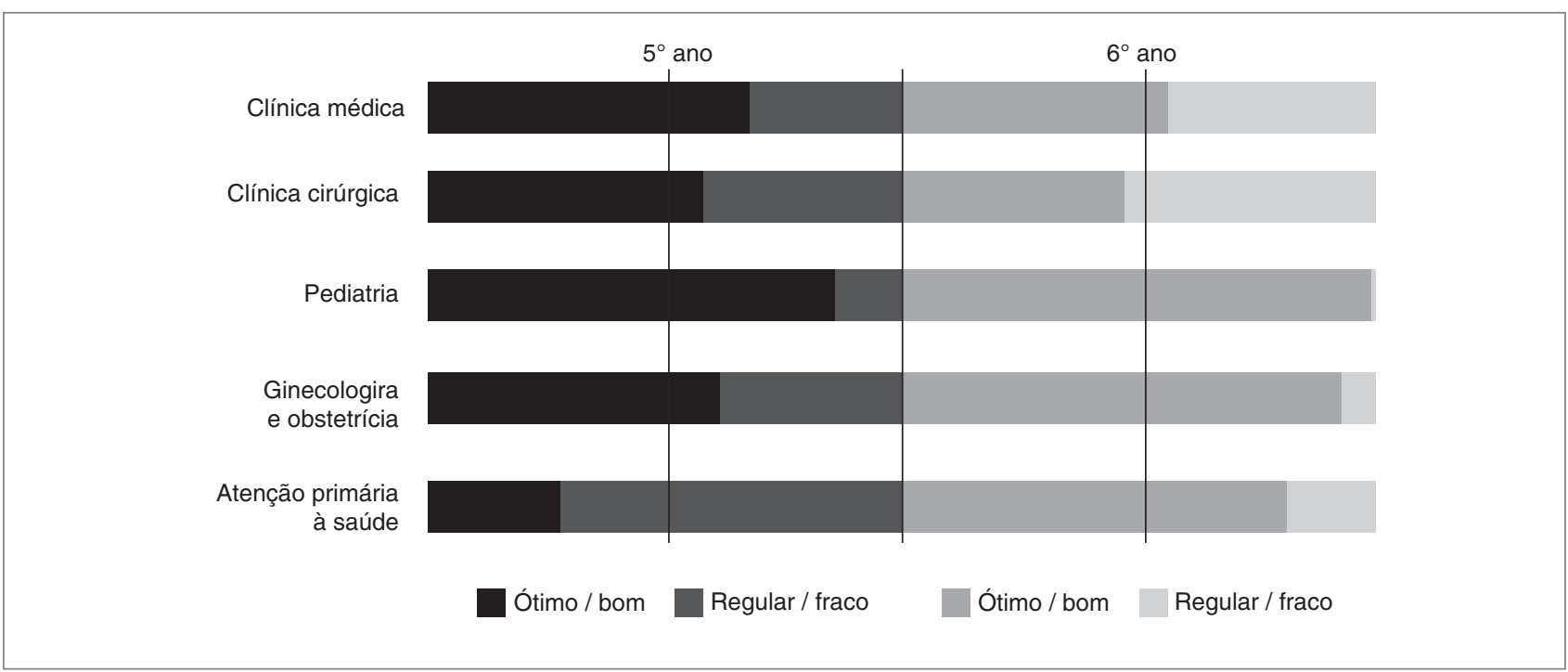

Figura 3: Matriz geral de avaliação do internato médico da Faculdade de Medicina do ABC, numa visão comparativa entre as disciplinas focando a qualidade do ciclo

Como espaço de prática, existem cinco estágios inseridos em uma parceria ensino/serviço em três municípios de São Paulo: São Bernardo do Campo, Santo André e São Caetano do Sul. Os alunos do quinto ano foram inseridos na estratégia de saúde familiar em três espaços de prática, sendo dois em São Bernardo do Campo (Parque Selecta e Orquídeas) e um em São Caetano do Sul (Moacir Galina).

Já os alunos do sexto ano contaram com uma abordagem de UBS no modelo tradicional, com dois espaços de prática: um em São Bernardo do Campo (Santa Terezinha) e o outro em Santo André (Centro de Saúde Escola Capuava). As diferenças entre o $5^{\circ}$ e $6^{\circ}$ anos levam a uma discussão com base nos dados obtidos. A percepção do Ciclo de Atenção Primária à Saúde e a sua inserção no internato médico é considerado satisfatório, no entanto, quando avaliados os dados do quinto e sexto anos separadamente, se observa uma discrepância entre os resultados.

A tal fator acrescentam-se as diferentes características do ensino durante o internato, na Faculdade de Medicina do ABC, em que o ciclo de Atenção Primária à Saúde no quinto ano tem como cenário de prática a Estratégia de Saúde de Família, enquanto o sexto ano baseia-se em uma UBS tradicional.

Nesse contexto, os alunos do sexto ano do internato de Medicina da FMABC têm como principal cenário de prática unidades-escolas, e são acompanhados por preceptor, podendo ser um médico especialista da unidade e/ou um docente universitário, cuja atividade é realizada por meio de parcerias entre a instituição de ensino e a prefeitura municipal. Os alunos participam do programa no bairro Capuava, no município paulista de Santo André desde 1986. A unidade dispõe de uma atividade de preceptoria composta na maioria por professores da FMABC. Logo, o envolvimento e a sensibilização desses profissionais se deram de maneira diferenciada, pois o docente que ministra aula na instituição em outros espaços da grade curricular faz a supervisão do estágio do internato. O vínculo prévio entre alunos e professores permite a valorização dos preceptores, mais experientes no que diz respeito à relação ensino/ aprendizagem e na rotina de integração entre ensino e serviço.

Na UBS do bairro Santa Terezinha, no município paulista de São Bernardo do Campo, os preceptores locados para orientação nessa unidade foram selecionados de acordo com um perfil estabelecido em 2007, ano de abertura da UBS em uma parceria com o Pró-Saúde, que permitiu a integração entre ensino/serviço, os profissionais/preceptores não eram docentes, mas egressos da instituição.

Nas unidades com ESF, foi desenvolvido outro modelo de sensibilização dos profissionais responsáveis pela execução da preceptoria. A primeira unidade de ESF inserida no internato foi a de Orquídeas, no município de São Bernardo do Campo, em que um médico foi sensibilizado com o projeto e recebeu os alunos.

A unidade de ESF do Parque Selecta, no município de São Bernardo do Campo, também foi beneficiada com a parceria com o Pró-Saúde, onde a maioria dos profissionais era formada por egressos da FMABC e ex-residentes da especialidade Medicina de Família e Comunidade. Essa unidade, com suas equipes de saúde da família, tinha um perfil pré-selecionado com profissionais capacitados e com características necessárias para desempenhar as atividades enfatizadas pelo programa.

No município de São Caetano do Sul, a secretaria de saúde selecionou a unidade de Estratégia de Saúde da família Moacir Galina, no bairro Cerâmica. Assim como no projeto da unidade Orquídeas, somente um profissional demonstrou interesse pelo projeto.

A atividade de preceptoria atrelada a demanda da comunidade e as metas propostas pelo SUS são fatores de tensão e estresse para esse profissional, que, para conseguir conciliar supervisão desses alunos e manter atividades que correspondam a um planejamento que cumpra os objetivos das diretrizes curriculares nacionais, acaba por ter um desgaste local importante na tentativa de manter, por exemplo, uma agenda compatível com supervisão de alunos da graduação e a demanda dos usuários da rede de saúde básica ${ }^{11}$. 
O estudo realizado por Pinto et $a l .{ }^{4}$ demonstra que os alunos que foram inseridos no internato na estratégia de saúde da família, colocam como críticas ao estágio aspectos que vêm de encontro ao modelo de formação do curso médico, em que os alunos estão acostumados a atuar como expectadores no processo de ensino e aprendizagem e receberem todas as coordenadas no período de estágio. Esse relato vem de encontro com os dados obtidos na percepção dos alunos nos quesitos teóricos do quinto ano inseridos no contexto da ESF (Figura 1). Tal fato demonstra uma dificuldade pelos alunos em assumir um grau de autonomia frente ao estágio, sendo o cenário de prática ideal no CAPS, um cenário onde deveriam ser atores principais e se propor a organizar o próprio tempo e buscar situações favoráveis ao seu aprendizado.

Uma situação oposta ocorre com os alunos do sexto ano. Todos os aspectos teóricos foram avaliados como ótimo e bom, pois, por estarem diante de uma estrutura mais conhecida, como citado anteriormente, e as atividades realizadas eram previamente estruturadas, os preceptores eram, na maioria, professores da instituição. O fator improviso e a solução de problemas não estavam, na maioria das vezes, envolvidos.

Quando analisamos os itens dos Aspectos práticos (Figura 2) e observamos os resultados do quinto ano, temos dois quesitos com resultado "Otimo + Bom". No primeiro, os alunos avaliam a sua dedicação durante o ciclo. O segundo aborda a orientação para a relação médico e paciente. Os demais itens avaliados demonstram pontos de tensão, considerados velhos conhecidos pelas dificuldades do processo de ensino e serviço, acompanhamento pelos preceptores, relação entre preceptor e aluno e se a clientela atendida foi suficiente para o aprendizado. Segundo Pinto et al. ${ }^{4}$, o papel do preceptor local merece um destaque importante, pois este profissional possui seus deveres e obrigações pelo trabalho de ESF, a ainda tem que conciliar a delicada tarefa de inserir os alunos do internato, com todas as suas expectativas e preconceitos, ao papel de mediador entre a equipe de saúde, secretaria municipal, e gestor local, com metas e objetivos de programas estabelecidos. "É dele a responsabilidade pela condução desse processo complexo, a qual irá possibilitar ou não a realização de todo o seu potencial"4.

Os alunos do sexto ano tiveram todos os itens dos aspectos práticos como ótimo ou bom. Esse preceptor é na sua maioria docente das disciplinas de Clínica médica, Ginecologia e Obstetrícia e Pediatria e que, juntos, integram o ciclo de Atenção Primária à Saúde neste período. Isso funciona como um facilitador quanto às expectativas e preconceitos dos alunos e não coloca este profissional com todas as demandas do outro preceptor da ESF. Os cenários de práticas são UBS tradicionais e "acostumadas" a receber alunos de vários estágios da graduação médica, além da experiência desse preceptor em organizar um programa pedagógico a contento com os objetivos do ciclo de internato.

$\mathrm{Na}$ discussão sobre o ensino-aprendizagem do aluno no internato de Medicina com base na problematização do cotidiano, Pinto et al. destacam que esse processo em serviços apresenta aspectos muito diferenciados daqueles efetuados em salas de aulas. Essa diferenciação ocorre tanto nos planos das relações quanto no plano dos conteúdos, onde se integram aos de caráter técnico-informativos. Nesse processo diferencia-se da educação formal, não se restringindo à transmissão de saberes, mas colocando-se como mediação de projetos contraditórios em construção ${ }^{4}$.

Em um estudo que discute as diretrizes para o ensino na Atenção Primária à Saúde (APS) na graduação em Medicina, Demarzo et al. ${ }^{5}$ afirmam que os estudantes são favorecidos ao lidar com diferentes aspectos da vida e seus ciclos, na sua complexidade clínica e cultural. Isso é possível devido à atuação em relação ao indivíduo e ao coletivo de forma contextualizada à realidade local; além de contribuir para o desenvolvimento de uma prática clínica integra$\mathrm{da}$, possibilitando a interdisciplinaridade.

Dermazo et al..$^{5}$ destaca que, ao final do sexto ano, o estudante de Medicina deve ter como competência três dimensões de forma integrada: "conhecer e utilizar a abordagem clínica centrada na pessoa integrada, complexa, interdisciplinar, longitudinal e resolutiva, utilizando as evidências científicas como ferramenta e suporte, mas singularizando o processo; estabelecer o primeiro contato com os pacientes, reconhecendo as incertezas no cotidiano da prática clínica da APS; desenvolver e aplicar a consulta de médico de família e comunidade para promover a relação médico-paciente; relacionar os processos específicos de decisão com a prevalência e a incidência das doenças na comunidade; reunir e interpretar seletivamente a informação recolhida na anamnese, no exame objetivo e nos exames complementares, e aplicá-la a um plano de ação adequado em colaboração com o paciente; manejar simultaneamente múltiplas queixas e patologias", além de promover a saúde e o bem-estar, conciliando as necessidades de cada paciente e as de saúde da comunidade ${ }^{5}$.

Foi realizado um estudo em $2009^{12}$ no curso de medicina na FMABC com o objetivo de avaliar a percepção dos egressos, quanto a Atenção Primária à saúde e também para avaliar o curso de graduação como um todo, sendo perguntado sobre as áreas de Pediatria, clínica médica, clínica cirúrgica, ginecologia e obstetrícia e saúde coletiva. Ao todo, 79,6\% dos egressos consideraram como Excelente e Bom a área da Pediatria, $75,1 \%$ a de ginecologia e obstetrícia, $66,5 \%$ a de clínica medica, $65,2 \%$ a de cirurgia, $24,3 \%$ a de saúde coletiva. Quanto à percepção sobre APS; $80 \%$ consideraram a APS como "a principal porta de entrada do SUS"; $14 \%$ caracterizaram que a APS "baixa complexidade tecnológica e pelo baixo custo operacional, lidando com problemas de saúde mais simples"; e 3\% caracterizaram "pela intervenção em aspectos biológicos e sociais da vida dos pacientes, na medida em que lida com problemas complexos".

Conclui-se que a formação de um profissional capaz de tomar iniciativas e solucionar problemas vai na direção contrária do modelo tradicional de ensino e aprendizagem do curso médico, em que ser passivo quantos aos aspectos do conhecimento e ter foco 
na memorização é a principal ferramenta pedagógica utilizada, deixando de lado processos essenciais de aprendizagem como, por exemplo, conjugar um método indutivo de conhecimento ao processo dedutivo ${ }^{13}$, onde a vivência dos alunos deveria servir de base para uma análise dos seus conhecimentos teóricos e a aplicação na prática.

Baseado neste estudo, há indícios de que os estudantes validaram a implementação do CAPS no curso de Medicina, mas ainda restam desafios e tensões quanto a essa estruturação e aceitação por parte dos estudantes da Estratégia de Saúde da Família como cenário de ensino apropriado para seu aprendizado.

\section{AGRADECIMENTOS}

Às professoras Simone Holzer e Ieda Cabral da Faculdade de Medicina do ABC.

\section{REFERÊNCIAS}

1. Machado MFAS, Monturo EMLM, Queiroz DT, Vieira NFC, Barroso MGT. Integralidade formação de saúde, educação em saúde e as propostas do SUS: uma revisão conceitual. Ciênc Saúde Coletiva. 2007;12(2):335-42. http://dx.doi.org/10.1590/S1413-81232007000200009

2. Starfield B. Atenção primaria: equilíbrio entre necessidades de saúde, serviços e tecnologia. Brasília: Unesco; Ministério da Saúde; 2002; p.726

3. Franco TB, Magalhães Júnior HM. Integralidade na assistência a saúde: a organização das linhas do cuidado. In: Merthy EE, Magalhães Júnior HM, Rimoli J, Franco TB, Bueno WS. O trabalho em saúde: olhando e experienciando o SUS no cotidiano. São Paulo: Hucitc; 2007; p.125-33.

4. Pinto LLS, Formigli VLA, Rêgo RCF. A dor e a delícia de aprender com o SUS: integração ensino-serviço na percepção dos internos em Medicina social. Rev Baiana Saúde Pública. 2007:31(1):115-33.

5. Demarzo MMP, Almeida RCC, Marins JJN, Trindade TG, Anderson MIP, Stein AT, et al. Diretrizes para o Ensino na Atenção Primária à Saúde na Graduação em Medicina. Rev Bras Educ Méd. 2012;36(1):143-8. http://dx.doi.org/10.1590/S0100-55022012000100020

6. Ferreira RC, Fiouni VML, Crivelaro E. Formação profissional no SUS: o papel da Atenção básica em saúde na perspectiva docente. Rev Bras Educ Med. 2010;34(2):207-15. http://dx.doi.org/10.1590/S0100-55022010000200004

7. Ceccim RB, Feuerwerker LCM. O quadrilátero da formação para a área da saúde: ensino, gestão, atenção e controle social. Physis. 2004; 14(1):41-65.

http://dx.doi.org/10.1590/S0103-73312004000100004
8. Merhy EE. Um dos grandes desafios para os gestores do SUS: apostar em novos modos de fabricar os modelos de atenção. In: Merhy EE, Magalhães Júnior HM, Rimoli J, Franco TB, Bueno WS. O trabalho em saúde: olhando e experienciando o SUS no cotidiano. São Paulo: Hucitec; 2003; p. 15-35.

9. Brasil. Ministério da Saúde. [Internet]. Programa nacional de reorientação da formação profissional em saúde Pró-Saúde. Disponível em: http://bvsms.saude.gov.br/bvs/publicacoes/pro saude_cgtes.pdf. Acesso em: 4 ago. 2007.

10. Marcondes E, Mascaretti LAS, Okay Y, Godoy GR. O desempenho do Departamento de pediatria da Faculdade de Medicina da Universidade de São Paulo no internato de 2001. Pediatria 2003;25(1/2):11-21.

11. Marin MJS, Oliveira MAC, Otani MAP, Cardoso CP, Moravcik MYAD, Conterno LO, et al. A integração ensino-serviço na formação de enfermeiros e médicos: A experiência da FAMEMA. Ciênc Saúde Coletiva. 2014:19(3):967-74

http://dx.doi.org/10.1590/1413-81232014193.09862012

12. Castellanos MEP, Silveira AFMH, Martins LC, Nascimento VB, Silva CS, Bortollette FHB, et al. Perfil dos egressos da Faculdade de Medicina do ABC: o que eles pensam sobre atenção primária em saúde? Arq Bras Ciênc Saúde. 2009;34(2):71-9

http://dx.doi.org/10.7322/abcs.v34i2.130

13. Garcia MAA. Saber, agir e educar: o ensino-aprendizagem em serviço de saúde. Interface. 2001;5(8):89-100. http://dx.doi.org/10.1590/S1414-32832001000100007 